\title{
Programming of Transcription and HPA Responses to Stress
}

\author{
Robert Skopec* \\ Dubnik, Slovakia, AXON, Researcher-analyst \\ *Correspondence to: Robert Skopec, Dubnik, Slovakia, AXON, Researcher-analyst; E-mail: zxcbnvm7@gmail.com
}

Received: December 01, 2016; Accepted: December 08, 2016; Published: December 13, 2016

\begin{abstract}
The signaling pathways link neuronal activity to transcription, revealing both the transcription factors that mediate this process and the neuronal activity-regulated genes. The neuronal activity regulates a complex program of gene expression involved in many aspects of neuronal development. Human genetic studies have revealed that the disruption of the activity-regulated gene expression program in humans gives rise to neurological disorders. Social states can affect health in further life. It is a completely revolutionary idea. Stress changes methylation and influence the whole life.
\end{abstract}

\section{Introduction}

The central aim is to formulate results based on studies in the fields of neurobiology and genetics to understand more human behavior at the level of neuropsychology. We have now a detailed molecular mechanism by which is possible to understand why social states can affect health in further life. It is a completely revolutionary idea.

The cellular and molecular mechanisms underlie to the experiencedriven changes in neural connectivity. Sensory experience results in neurotransmitter release at synapses within a neural circuit and leads to membrane depolarization, calcium influx into individual neurons. Which triggers a wide variety of cellular changes with these neurons capable of altering synaptic connectivity of the circuit. Changes such as the activation of calcium-sensitive signaling cascades lead to posttranslational modifications of proteins, at the regulation of mRNA translation [1]. It's resulting in the production of new proteins locally at the sites of calcium entry and play critical roles in altering synaptic function in a synapse-specific manner.

\section{Materials and Methods}

We have used the studies cited in the references to make a review from the latest results at the field of neurobiology, genetics, and neuropsychology to analyze what are the mechanisms regulating human behavior at neural and psychological level under conditions of stress. We try to formulate how sensory information influences response behavior by semi-analytical, information theoretical, statistical and neuropsychological methods.

To understand more human behavior in the psychological conditions of stress we must start from the underlying principles of neurobiology and genetics. It can be done by the method of relating neurobiological models to behavioral models of signaling pathways.

\section{Calcium influx can alter cellular function by activating new gene transcription}

Calcium influx into the postsynaptic neuron can alter cellular function by activating new gene transcription. Calcium influx activates a number of signaling pathways converging on transcription factors within the nucleus, which in turn control the expression of a large number of neuronal activity regulated genes. Signaling pathways mediate activity-dependent transcription in experience-dependent neural development and plasticity. This neuronal activity regulates by the signal transduction pathways the activity-dependent gene expression program. On the other side, neuronal activity-regulated genes showing how this activity-regulated program controls neuronal development $[1,2]$.

The $c$-fos mRNA is induced by synaptic activity resulting from sensory experience due the Fos protein with Jun family members comprised the AP- 1 transcriptional complex, which is critical for the organism's adaptive responses to experience. A brain-specific deletion of the $c$-fos gene displays deficits in synaptic plasticity and defects in learning and memory. Loss of Fos-dependent transription gives raise to additional behavioral deficits. [3]

The activity-regulated transcriptional program uncovered a mechanism by which calcium-dependent gene induction alters the function of specific synapses. The translation of select mRNAs can occur at individual synapses through the actions of microRNAs (miRNAs) which inhibit the translation of mRNAs having nucleotide sequences closely matching the miRNAs. The level of miR-134 is increased by neuronal activity. The miRNA could be a component of the local mRNA translation machinery allowing proteins to be translated in a synapse-specific manner. This transcriptional program is critical in coordinating both dendritic and synaptic remodeling.

\section{The transcriptions of $c$-fos and other immediate early genes}

The transcriptions of $c$-fos and other immediate early genes (IEGs) increases in many cells of the body in response to extracellular factors inducing proliferation or differentiation of the cells. IEGs mediate cellular responses to changes in the cell's environment. Recent studies have identified a subset of genes that is activated specifically in response 
to excitatory synaptic transmission that triggers calcium influx into the postsynaptic neuron. One gene is specifically induced by neuronal activity in neurons: $b d n f$ encodes a neurotrophin important in neural development. The level of the $b d n f$ mRNA increases in neurons in response to physiological stimuli, such as fear conditioning and seizure induction. The induction of the $b d n f$ mRNA is due to an increase in transcription of the $b d n f$ gene [4].

Transcripts of these promoters splice from their first exon to a common downstream exon, which contains the entire open reading frame encoding the BDNF protein. This diversity could explain how BDNF can control such a large number of distinct processes during nervous system development. Neuronal activity sharply increases the rate of transcription initiation with most transcripts ending within the central intron. These coordinate transcriptional events rapidly convert a constitutive gene to an IEG and regulate the expression of functionally different Homer 1 proteins. The short forms modulate the properties of the long forma and are critically involved in activitydependent alterations of synaptic structure and function. [1-3]

\section{The switch from constitutive to activity-dependent expression}

The switch from constitutive to activity-dependent expression entails intronic to exonic sequence conversion, transcript termination within the central intron of the Homer 1 gene. Homer proteins play key roles in signal transduction in the brain [1-3]. Hypothalamicpituitary-adrenal (HPA) responses to stress suggesting a causal relation among epigenetic state, glucocorticoid receptor (GR) expression and the maternal effect on stress responses in the later offspring. There are increasing number of the results confirming that an epigenomic state of a gene may be established through forms of an environmental and programming and this is potentially reversible. [4]

Variations in maternal behavior are connected with development of individual differences in behavioral and HPA responses to stress in the offspring. They serve as a mechanism for the nongenomic transmission of individual differences in stress reactivity across generations. Recent findings suggest that the mechanisms of these maternal effects, or other forms of environmental programming, remain sustained over the lifespan. [5]

Maternal behavior in the rat permanently alters the development of HPA responses to stress through tissue-specific effects on gene expression. The magnitude of the HPA response to stress is a function of hypothalamic corticotropin-releasing factor (CRF) release, thus activating the pituitary-adrenal system. There are also some modulatory influences, like glucocorticoid negative feedback, which inhibits CRF synthesis and release, dampening HPA responses to stress. [4]

\section{Epigenetic programming}

The changes in Avp expression were restricted to the parvocellular subpopulation of neurons in the hypothalamic paraventricular nucleus $(\mathrm{PVN})$ in those neurons that drive the HPA axis. Research data verify the critical role of arginine vasopressin (AVP) in driving the disturbed endocrine phenotype in stressed mice. This hypothesis was supported by the observation that the methyl CpG-binding protein 2 (MeCP2) phosphorilation was prominently increased in parvocellular AVPexpressing neurons in the PVN. Phosphorilation of MeCP2 at S438 is critical for MeCP2 function as a reader and interpreter of the DNA methylation signal at the Avp enhancer. MeCP2 serves as an epigenetic integration platform on which synergistic cross-talk between histone deacyclation, K3K9 methylation and DNA methylation act to confer gene silencing. [1,2,4]

Research data suggests that stress tilts the balance toward persistent hypomethylation and Avp overexpression by inducing reductions in $\mathrm{MeCP} 2$ binding. Phosphorilation of MeCP2 appears to be a carrier of experience-driven changes in gene expression, as a important mediator of the persistent effects of stress. By DNA methylation, there are evidence for postmitotic epigenetic modifications in neuronal functions. Modifications can facilitate or disfavor physiological and behavioral adaptations [2]. Epigenetic marks and their initiators, mediators and readers (MeCP2) bring new evidences for understanding the molecular basis of stress-related disorders of the brain.

\section{Glucocorticoid programming}

Genetic background might predispose to early-life events as maternal care, which can change the genetic profile through epigenetic signaling pathways. The programming effect of maternal behavior is associated with a single gene: the glucocorticoid (GR) gene. The offspring of caring mothers had higher hippocampal GR expression, owing to demethylation of a cysteine residue at the 5'NGF1A binding region in the exon 1, promoter [1]. Corticosteroids operate in both stress-system modes through mineralcorticoid (MR) and GR receptors co-expressed in the neurons of limbic structures. MR acts in the appraisal process and the onset of the stress response. GR is only activated by large amounts of corticosteroid, terminates the reactions to competition (the stopping rule). GR also promotes memory storage in preparing for future events $[4,6]$.

\section{Behavioral programming}

In vivo studies suggest that the effect of maternal behavior on GR gene expression is accompanied by an increased hippocampal expression of nerve growth factor-inducible protein A (NGFI-A). The non-coding exon 1 region of the hippocampal GR includes a promoter region, exon $1_{7}$, containing a binding site for NGFI-A. Splice variants of the GR mRNA containing the exon $1_{7}$ sequence are found predominantly in the brain.

Use of promoter is enhanced as a function of maternal care, what explain the increased GR expression in the neonate. Maternal care alters DNA methylation of the GR exon $1_{7}$ promoter, and these changes are stably maintained into adulthood, associated with differences in GR expression and HPA responses to stress. Variations in maternal care directly alter the methylation status of the exon $1_{7}$ promoter of the GR gene. DNA methylation pattern can be established also through a behavioral programming without germ line transmission [4]. Postnatal de novo methylation of the Hoxa5 and Hoxb5 genes in development was documented also in another study [7]. 
Thus, maternal programming of the exon $1_{7}$ GR promoter involves DNA methylation, histone H3-K9 acetylation and alterations in NGFI-A binding $[4,6]$. The afferent input from limbic networks converts purely psychological stress reactions to the HPA axis. Above interplay of limbic inputs from the hippocampus, amygdala and prefrontal cortex with HPA axis activity may lead to a vulnerable phenotype for mental illness [4, 5].

\section{Environmental programming}

We have now evidence that maternal behavior produces stable alterations of DNA methylation and chromatine structure, providing a mechanism for the long-term effects of maternal care on gene expression in the offspring. Such a gene-environment interactions during development result in the sustained environmental programming of gene expression and function of defensive responses through increased HPA activity over the lifespan [4, 5]. Natural selection shaped offspring to respond to subtle variations in parental behavior as forecast of the environmental conditions. They serve as a major source of epigenetic variations in gene expression and mediating such maternal effects. Effects on chromatine structure serve as an intermediate process imprinting dynamic environmental experience on the fixed genome with stable variations in phenotype $[1,2,5]$.

\section{Environment-assisted invariance}

The state of composite object (consisting of the system $S$ and the environment $E$ ) can be ignorant of the state of $S$ alone. Environmentassisted invariance, or envariance based on symetry allows observer to use perfect knowledge of $S E$ as a proof of his ignorance of $S$ : when a $U_{S}$ acting on $S$ alone, can be undone by a transformation acting solely on $E$, and the joint state of $S E$ is unchanged. This state is said „envariant" with respect to $U_{S}$. Envariant properties not belong $S$ alone. Entanglement between $S$ and $E$ enables envariant and implies ignorance about $S$. Envariance is associated with phases of the Schmidt decomposition of the state representing SE. It anticipates the consequences of environment - induced superselection („einselection“) of the preferred set of pointer states, they remain unperturbed to immersion of the system in the environment. The state of combined SE expressed in the Schmidt form is: $|\psi S E\rangle=\sum_{k=1}^{N} \alpha_{k}\left|\delta_{k}\right\rangle\left|E_{k}\right\rangle$. Schmidt states are in an intimate relationship with the pointer states and have been regarded as „instantaneous pointer states“ [8]. Quantum Darwinism brings new focus on the environment as a communication channel. This explains the emergence of objectivity. Even hazy environment will communicate a very clear image [9].

\section{Adaptational programming}

Limbic pathways activated by psychological stressors of competition are parts of the afferent pathways activating the CRH neurons in the PVN. The interface between incoming sensory information and the appraisal is converted by limbic brain structures (the hippocampus, amygdala and prefrontal cortex-PFC).
Not only homeostatic disturbance, but purely psychological code can determine the stress response to competition. Its determinants include the ability predict upcoming events and getting control over the situation. The adaptive competition stress-related processes take place in limbic brain regions. An inappropriate response to the winner-take-all instabilities (WTAIs) produces a vulnerable phenotype leaving genetically predisposed individuals at an increased risk of stress-related brain disorders. $[5,10]$.

Multiple peaks of activity appear simultaneously within a single frontal or parietal region, they compete against each other through inhibitory antagonism. This can be seen in biased competition mechanism of visual attention. During colour-cue period preferring the given colour pushes group of cells towards stronger activity than others and causes the competition in dorsal premotor cortex (PMd) to become unbalanced, because one peak increases its activity, while the other is suppressed. Since neural activities are noisy, competition between distinct peaks of activity cannot follow a simple winnertake-all rule, or random fluctuations will determine the winner each time. If activity of a given choice becomes sufficiently strong, than it should be allowed to suppress its opponent and conclusively win the competition. But the cost of reinstating homeostasis also might become too high, causing through WTAIs an allostatic load with increased risk of mental illness [5,11-19].

\section{Dynamics of the winner-take-all instability}

To derive an equation for the dynamics of the winner-take-all instability, we express the dynamical variables as $x=x_{S S}+x_{c r} Y(T)+\ldots$ where $Y$ represents the slow dynamics along the critical eigenvector and $T$ is a slow time scale. The reflection symmetry of the system implies the dynamics of $Y$ should be invariant under the transformation $Y \rightarrow-Y$ and this switches the identity of $x_{1}$ and $x_{2}$. The increase in input $I$ is common to both $x_{1}$ and $x_{2}$ leads to the developing decision in the winner-take-all system and is thus the bifurcation parameter. The linear growth rate of the spontaneous state must be proportional to the difference between the presynaptic input and the value of the input at the bifurcation with an unknown prefactor, i.e. $\mu\left(I-I_{c c}\right)$. The difference in inputs $I_{1}-I_{2}$ breaks the reflection symmetry thereby introducing a constant term which, to first approximation, must be proportional to that difference with an unknowvn prefactor, i.e. $\eta\left(I_{1}-I_{2}\right)$. These two facts, coupled with the reflection symmetry, lead to the form of the equation describing the time evolution of $Y: \delta_{T} Y=\eta\left(I_{1}-I_{2}\right)+\mu\left(I-I_{c r}\right) Y+\gamma Y^{3}$, where $I=I_{c r}$ only when $\alpha=\beta$ identically, i.e. at point of instability, and $\delta_{T}$ is a time derivative with respect to the slow time $T$. For $I_{1}-I_{2}$ the equation is invariant under $Y \rightarrow-Y$ as it should be, $Y^{3}$ is the lowest order nonlinearity which obeys reflection symmetry. For more complex systems, which exhibit winner-take-all behavior, above euation captures the qualitative dynamics of the system near the bifurcation in general [20].

\section{Concluding Remarks}

During adaptation sensory experience driven changes in neural connectivity, transcription, and HPA axis responses to stress are complex and multifactorial: they cannot be attributed to mutations in single gene, or to a single external event, but rather, result from 
the concerted actions of many subtle genetic polymorphisms and external events, the effects of which might accumulate over time. Once traumatic life events, in combination with genetic disposition, have engrained long-lasting changes in MR and GR signaling, a vulnerable phenotype emerges [5].

DNA methylation is behind the changes associated with stress. It is based on differences in the gene encoding AVP, a hormone associated with mood and cognitive behavior. After stress, there was lover level of methylation in the regulatory region of the Avp gene in the brain. This hypomethylation was specific to a subset of neurons in the hypothalamic paraventricular nucleus - a brain area involved in regulating hormones linked to stress $[1,2]$.

The decreases in methylation in stressed subjects result from the inactivation of a protein $\mathrm{MeCP} 2$, involed in the start of the DNA methylation. It is a detailed molecular mechanism by which is possible to understand why social states as sensory experience can affect health in further life. It is a completely revolutionary idea [2]. Stress changes methylation and influence the whole life. Depression may be facilitated by a failure in competition to contain the biological stress response to challenge of unemployment at the time of the trauma, resulting in a cascade of alterations leading to recollections of the WTAIs, avoidance of the reminders to event and symptoms of hyperarousal [5]. From psychological and biological data we may hypothesize that the pathological mechanism of stress-related brain disorders depend on distress connected with inhibitory antagonism produced by winner-take-all instabilities. Mechanism is triggered by interactive behavior of an appraisal of unit $\mathrm{P}$ probabilities trade-off with environment. Stressors can kill with information itself through probabilities. Probabilities are the killer by information [9].

Sensory information itself, as first communication of diagnosis, may act as psychic stressor, psychological weapon (of mass destruction) due stress-related brain disorders [11-18]

It is well documented in recent large population-based study about men newly diagnosed with prostate cancer, they were at higher risk of cardiovascular events and suicide. The excess risks were highest during the first week after diagnosis, suggesting that stress of diagnosis itself plays a critical role. The emotional stress as an information itself caused a cardiovascular morbidity increase immediately after communication of the diagnosis [18].

Emotionally stressful competition events may lead to altered function of the heart, a stress-related left ventricular dysfunction [15]. Increased risk of myocardial infarction was documented following the Athen earthquake in 1983 [14]. Emotional stress brought on by viewing a World cup soccer match was reported to raise the risk for cardiovascular morbidity and mortality [17]. Being informed about diagnose of prostate cancer may also serve as a stressor of substantial weight. About $20 \%$ of the prostate cancer patients were reported as having no one to confide in Fall K, et al. [18]. On the basis of above results bring a hypothesis of the weights function in a framework of feedback paradigm as the psychological code. Possible mechanism may be the emotional shock caused by the information of diagnosis, anxiety, together with emotional isolation.

\section{References}

1. Flavell SW and Greenberg ME (2009) Signaling mechanisms linking neurona activity to gene expression and plasticity of the nervous system. Ann Rev Neurosci 2008:31:583-590. [Crossref]

2. Murgatroyd C, Patchev AV, Wu Y, Micale V, Bockmühl Y, et al. (2009) Dynamic DNA methylation programs persistent adverse effects of early-life stress. Nat Neurosci 12: 1559-1566. [crossref]

3. Bottai D, Guzowski JF, Schwarz MK, Kang SH, Xiao B, et al. (2002) Synaptic activity-induced conversion of intronic to exonic sequence in Homer 1 immediate early gene expression. $J$ Neurosci 22: 167-175. [crossref]

4. Weaver IC, Cervoni N, Champagne FA, D'Alessio AC, Sharma S, et al. (2004) Epigenetic programming by maternal behavior. Nat Neurosci 7: 847-854. [crossref]

5. de Kloet ER, Joëls M, Holsboer F (2005) Stress and the brain: from adaptation to disease. Nat Rev Neurosci 6: 463-475. [crossref]

6. Seck1 JR, Meaney MJ (2004) Glucocorticoid programming. Ann N Y Acad Sci 1032: 63-84. [crossref]

7. Hershko AY, Kafri T, Fainsod A, Razin A (2003) Methylation of HoxA5 and HoxB5 and its relevance to expression during mouse development. Gene 302: 65-72. [crossref]

8. Zurek WH (2002) arXiv: 0211.037 v1 [quant-ph]

9. Zwolak M, Quan HT, Zurek WH (2009) arXiv: 0904.0418v2 [quant-ph]

10. Cisek $P$ (2007) Cortical mechanisms of action selection: the affordance competition hypothesis. Philos Trans R Soc Lond B Biol Sci 362: 1585-1599. [crossref]

11. Meisel SR, Kutz I, Dayan KI, Pauzner H, Chetboun I, et al. (1991) Effect of Iraqi missile war on incidence of acute myocardial infarction and sudden death in Israeli civilians. Lancet 338: 660-661. [crossref]

12. Li J, Hansen D, Mortensen PB, Olsen J (2002) Myocardial infarction in parents who lost a child: a nationwide prospective cohort study in Denmark. Circulation 106: 1634-1639. [crossref]

13. Li J, Laursen TM, Precht DH, Olsen J, Mortensen PB (2005) Hospitalization for mental illness among parents after the death of a child. $N$ Engl J Med 352: 1190-1196. [crossref]

14. Katsouyanni K, Kogevinas M, Trichopoulos D (1986) Earthquake-related stress and cardiac mortality. Int J Epidemiol 15: 326-330. [crossref]

15. Wittstein IS, Thiemann DR, Lima JA, Baughman KL, Schulman SP, et al. (2005) Neurohumoral features of myocardial stunning due to sudden emotional stress. $N$ Engl J Med 352: 539-548. [crossref]

16. Schairer C, Brown LM, Chen BE, Howard R, Lynch CF, et al. (2006) Suicide after breast cancer: an international population-based study of 723,810 women. $J$ Natl Cancer Inst 98: 1416-1419. [crossref]

17. Wilbert-Lampen U, Leistner D, Greven S, Pohl T, Sper S, et al. (2008) Cardiovascular events during World Cup soccer. $N$ Engl J Med 358: 475-483. [crossref]

18. Fall K, Fang F, Mucci LA, Ye W, Andrén O, et al. (2009) Immediate risk for cardiovascular events and suicide following a prostate cancer diagnosis: prospective cohort study. PLoS Med 6: e1000197. [crossref]

19. Leor J, Poole WK, Kloner RA (1996) Sudden cardiac death triggered by an earthquake. $N$ Engl J Med 334: 413-419. [crossref]

20. Roxin A, Ledberg A (2008) Neurobiological models of two-choice decision making can be reduced to a one-dimensional nonlinear diffusion equation. PLoS Comput Biol 4: e1000046. [crossref]

Citation:

Robert Skopec (2016) Programming Of Transcription and HPA Responses to Stress. Cancer Stud Ther J Volume 1(2): 1-4 